\title{
Detection of individual prostate cancer via multiparametric magnetic resonance imaging in own material - initial experience
}

\author{
Paweł Porzycki, MD!, Ewa Ciszkowicz, PhD² \\ 'Department of Urology, Municipal Hospital Rzeszow, Rzeszow, Poland, ²Department of Biotechnology and Bioinformatics, \\ Faculty of Chemistry, Rzeszow University of Technology, Rzeszow, Poland
}

\begin{abstract}
Purpose: Multiparametric magnetic resonance imaging (mpMRI) is an evolving non-invasive imaging modality that increases the accurate localization of prostate cancer (PCa) at the time of MRI targeted biopsy, enhancing clinical risk assessment, and improving the ability to appropriately counsel patients regarding therapy.

Material and methods: A total of forty patients with prostate-specific antigen (PSA), mpMRI and Gleason score (based on MRI template-guided cognitive biopsy) results were analyzed in this study, with eight patients (20\%) diagnosed with PCa. The mpMRI was performed to facilitate the decision to perform prostate biopsy. Spearman's coefficient analysis was used to evaluate the relationships between characteristics. Diagnostic performance was assessed measuring the area under the curve (AUC) of the receiver operating characteristic (ROC) analysis. Diagnostic accuracy, sensitivity, and specificity were determined using the best cut-off on each ROC.

Results: Out of all the study group, $55 \%$ of patients were subjected to primary biopsy and $45 \%$ were directed to repeated TRUS-Bx with the suspicion of prostate cancer. Forty suspected lesions on MRI images were identified with $5 \%$ of PI-RADS $1,17.5 \%$ of PI-RADS $2,32.5 \%$ of PI-RADS $3,27.5 \%$ of PI-RADS $4(27.5 \%)$ and $17.5 \%$ of PI-RADS 5. The highest correlation was observed for mpMRI results and Gleason score with Spearman's coefficient equal to 0.41 (95\% CI: 0.104-0.646). ROC analysis revealed that mpMRI discriminates between directing the patients for prostate biopsy or active surveillance with AUC $=0.771(0.117,95 \%$ CI: 0.542-1.001).

Conclusions: Introducing pre-biopsy mpMRI into our contemporary PCa diagnosis pathway increased the diagnostic yield of transrectal biopsy by increasing the prostate cancer detection. This enabled the introduction of clinically significant prostate cancer (csPCa) treatment. mpMRI application also allowed biopsy to be avoided among patients with no csPCa.

Key words: mpMRI, PI-RADS, biopsy, prostate cancer, brachytherapy.

\section{Purpose}

Prostate cancer (PCa) is the most common type of cancer among men in Europe. Current recommendations for screening and diagnosis are based on prostate-specific antigen (PSA) levels and/or suspicious digital rectal examination (DRE) and/or imaging. Transrectal ultrasound-guided biopsy (TRUS-Bx) is now the standard of care. The contemporary random 10-12-core systematic biopsy strategy relies on sampling efficiency for cancer detection. Prostate biopsy is performed by either the transrectal or transperineal approach [1]. Although laterally directed cores within the peripheral zone increase detection, prostate cancers are frequently multifocal, small, intermingled with benign stroma, and not uniformly dis- tributed within the gland. Consequently, clinically significant cancers frequently go undetected when employing the standard 12-core biopsy template [1]. Conventional TRUS-Bx also leads to incorrect risk stratification of tumors as low volume or low grade. These clinically insignificant cancers are often identified by chance during a systematic biopsy, contributing, in part, to the problem of over-detection and over-treatment of low-risk and indolent PCa [2].

Advances in multiparametric magnetic resonance imaging (mpMRI) have demonstrated an improvement in detection and characterization of clinically significant prostate cancer (csPCa). mpMRI is increasingly used to localize suspicious areas that could be targeted by magnetic resonance imaging-targeted biopsies (MRI-TBx) [3]. 
Introducing mpMRI and MRI targeted biopsy as modalities to evaluate men at risk for prostate cancer may help in determining more efficiently which men need a prostate biopsy. MRI targeted biopsy enables more accurate sample collection, thereby allowing greater detection of clinically significant disease with fewer biopsy cores, more accurate risk stratification, and avoidance of the detection of indolent disease [4,5]. MRI targeted biopsy enables a better risk stratification among men who are subjected to active surveillance.

Once the prostate cancer is diagnosed, it can be radically treated by surgery (radical prostatectomy) or by irradiation therapy according to available guidelines or patient preferences. The recent developments in the field of irradiation technology have made radiotherapy one of the main local treatment methods for the prostate cancer patients. Brachytherapy low-dose-rate (LDR) or highdose-rate (HDR) is a standard primary treatment for low- or intermediate-risk PCa [6]. TRUS-Bx is routinely acquired for LDR prostate brachytherapy preplanning (pTRUS), although diagnostic mpMRI may serve this purpose as well [7]. Fredman et al. compared the predictive abilities of TRUS vs. MRI relative to intraoperative TRUS (iTRUS) to assess the role of mpMRI in brachytherapy preplanning. Shaaer et al. described the use of $\mathrm{mp}-$ MRI during prostate HDR brachytherapy planning [8]. These works suggest that mpMRI carries numerous diagnostic, staging and preplanning advantages that facilitate better patient selection and delivery of novel dose escalation and targeted therapy, with no additional surgical or anesthesia time $[7,8]$.

The group of patients who were diagnosed with the pre-biopsy mpMRI (PB-mpMRI) tool for the detection of PCa is presented in the article. The aim of this study was to assess the diagnostic value of PB-mpMRI among the group of patients referred for a primary and secondary biopsy.

\section{Material and methods}

In the period between November 2018 and May 2019, a group of 40 consecutive patients, selected in a single urological institution, who were considered for a prostate biopsy with a suspicion of PCa, was evaluated. The contrast-enhanced mpMRI test was performed to facilitate the decision to perform prostate biopsy among men with elevated PSA levels (> $4 \mathrm{ng} / \mathrm{ml}$ ), abnormal DRE or family history of PCa. The men with previous negative biopsies (two or more biopsies before), among whom the suspicion of prostate cancer was still present, were qualified for another biopsy and the MRI-TBx procedure was performed. In areas of suspicion, identifying hidden diseases by the MRI-TBX procedure is advantageous. Radiology technicians performed mpMRI scans according to the standardized protocol. All men underwent $1.5 \mathrm{~T}$ (31 patients $-77.5 \%$ ) or $3 \mathrm{~T}$ (9 patients $-22.5 \%$ ) mpMRI. The evaluation of MRI data was carried out in accordance with the Prostate Imaging-Reporting and Data System (PI-RADS) v.2 and improved by a sectoral map. Three senior radiologists, with relevant experience in Prostate Imaging-Reporting and Data System v.2 (PI-RADS v.2) reporting, evaluated suspicious lesions using T2-weight- ed imaging, diffusion-weighted imaging (DWI) and dynamic contrast-enhanced MRI [9]. The radiologists had access to clinical information and assigned a PI-RADS v.2 suspicious result to each region of interest (ROI). The ROI found on the MRI scan diagram as depicted by the radiologist (suspicious lesions) was projected (cognitively) prior to the procedure. We could not evaluate inter-reader variability as two different readings were not available. PI-RADS > 3 were considered suspicious. Patients with mpMRI lesions (PI-RADS 4-5) underwent cognitive targeted (COG-Bx) plus systematic TRUS-Bx. We defined lesion-specific csPCa as Gleason score $>7(3+4)$ [10]. The patients with PI-RADS 1-3 on mpMRI avoided immediate biopsy; they are under the standard care and supervision. The procedure was performed with prophylactic intravenous antimicrobial cover (ciprofloxacin). The patients were also instructed to continue oral antibiotics for another 4 days. We investigated the impact of introducing mpMRI on the diagnostic yield of transrectal prostate biopsy (TRUS-Bx).

Data preprocessing and statistical analyses were performed using Analyse-it for Microsoft Excel Method Validation Edition (version 2.20) and STATISTICA v.12 (StatSoft, OK, USA) software. The Mann-Whitney method was applied to analyze the statistical difference between groups of patients and Spearman rank correlation was used to analyze the data. Statistically significant differences were set at $p<0.05$ using the two-sided test. Receiver operating characteristics (ROC) curves and the area under the ROC curves (AUC) were calculated to determine the potential of mpMRI and to discriminate between the patients who should undergo prostate biopsy and those who may be under constant observation (active surveillance), to reduce the false-negative rate of biopsy and avoid detection of low-grade disease. ROC curves were also assessed to determine the potential of physiological characteristics to discriminate between PCa and no-PCa (not diagnosed for prostate cancer) patients.

\section{Results}

Out of 40 consecutive men included in the study group, 55\% were subjected to primary biopsy and $45 \%$ were directed to redo TRUS-Bx with the suspicion of prostate cancer. The median age of all patients was 66 . The general median PSA was $7.1 \mathrm{ng} / \mathrm{ml}$ and median PSA density (PSAD), calculated on the basis of prostate volume obtained by mpMRI, was 0.181 . We defined 40 suspected lesions on MRI images - 2 lesions PI-RADS 1 (5\%), 7 lesions PI-RADS 2 (17.5\%), 13 lesions PI-RADS 3 (32.5\%), 11 lesions PI-RADS 4 (27.5\%) and 7 lesions were scored PI-RADS 5 (17.5\%). Within the group of patients with $\mathrm{PCa}$, cancer tissue was identified only in the peripheral zone, while in the no-PCa group $56.25 \%$ and $40.6 \%$ of altered prostate tissue was localized, respectively, in transitional and peripheral zones. The patient characteristics are shown in Table 1.

Two-sided Spearman's correlation test was conducted for relationships between mpMRI, PSA and PSAD within the entire study group (40 patients) and for Gleason score, mpMRI, PSA and PSAD within the PCa group 
Table 1. Patients' characteristics

\begin{tabular}{|c|c|c|c|c|}
\hline Characteristic & $\begin{array}{l}\text { All patients } \\
(n=40) \\
\end{array}$ & $\begin{array}{c}\text { After biopsy } \\
\text { with PCa }(n=8) \\
\end{array}$ & $\begin{array}{c}\text { After biopsy } \\
\text { without PCa }(n=2)\end{array}$ & $\begin{array}{l}\text { No biopsy } \\
(n=30)\end{array}$ \\
\hline $\begin{array}{l}\text { Median age } \\
\text { (range) }\end{array}$ & $\begin{array}{c}66 \\
(51-84) \\
\end{array}$ & $\begin{array}{c}67 \\
(51-74) \\
\end{array}$ & $\begin{array}{c}67.5 \\
(65-70) \\
\end{array}$ & $\begin{array}{c}66 \\
(53-84) \\
\end{array}$ \\
\hline $\begin{array}{l}\text { Median PSA (ng/ml) } \\
\text { (range) }\end{array}$ & $\begin{array}{c}7.1 \\
(0.49-22.13) \\
\end{array}$ & $\begin{array}{c}9.75 \\
(0.49-22.13) \\
\end{array}$ & $\begin{array}{c}9.5 \\
(4.01-15) \\
\end{array}$ & $\begin{array}{c}6.84 \\
(3.75-17.86) \\
\end{array}$ \\
\hline $\begin{array}{l}\text { Median PSAD (ng/ml/ml) } \\
\text { (range) }\end{array}$ & $\begin{array}{c}0.181 \\
(0.025-0.2) \\
\end{array}$ & $\begin{array}{c}0.21 \\
(0.025-0.461) \\
\end{array}$ & $\begin{array}{c}0.15 \\
(0.1-0.2) \\
\end{array}$ & $\begin{array}{c}0.173 \\
(0.048-1.2)\end{array}$ \\
\hline \multicolumn{5}{|l|}{ Gleason score } \\
\hline $7(4+3)$ & 2 & 2 & - & - \\
\hline $7(3+4)$ & 3 & 3 & - & - \\
\hline $6(3+3)$ & 3 & 3 & - & - \\
\hline \multicolumn{5}{|l|}{$\mathrm{MRI}$} \\
\hline 1 & 2 & 1 & 1 & 0 \\
\hline 2 & 7 & 0 & 0 & 7 \\
\hline 3 & 13 & 0 & 1 & 12 \\
\hline 4 & 11 & 3 & 0 & 8 \\
\hline 5 & 7 & 4 & 1 & 2 \\
\hline \multicolumn{5}{|l|}{ MRI (prostatic zone) } \\
\hline Peripheral & 20 & 7 & 1 & 12 \\
\hline Transitional & 18 & 0 & 0 & 18 \\
\hline No change & 2 & 1 & 0 & 1 \\
\hline
\end{tabular}

$P C a$ - identified prostate cancer, PSA - prostate specific antigen, MRI - magnetic resonance imaging

Table 2. Results of Spearman's correlation analysis (significance levels for each correlation pair are represented by italics)

\begin{tabular}{lcccc} 
Correlation pairs & Group size & Spearman's rs & $95 \% \mathrm{Cl}$ & $\begin{array}{c}p \text {-value } \\
(n=0.05)\end{array}$ \\
\hline mpMRI, PSA & 40 & 0.378 & $0.066-0.623$ & $0.0162^{\star \star}$ \\
\hline mpMRI, PSAD & 40 & 0.261 & $-0.065-0.536$ & $0.1039^{\star}$ \\
\hline mpMRI, Gleason score & 8 & 0.41 & $0.104-0.646$ & $0.0085^{\star \star \star}$ \\
\hline PSA, Gleason score & 8 & 0.237 & $-0.09-0.518$ & $0.1409^{\star}$ \\
\hline PSAD, Gleason score & 8 & 0.33 & $0.011-0.588$ & $0.0378^{\star \star}$
\end{tabular}

${ }^{*} p<0.1,{ }^{* *} p<0.01,{ }^{* * *} p<0.001$

(8 patients). Obtained Spearman's correlation coefficients $\left(r_{s}\right)$ values significant in the range $0.001<p<0.1$ are presented in Table 2.

ROC analyses were performed to evaluate the capability of mpMRI to discriminate between direction of patients for prostate biopsy or active surveillance (Table 3, Figure 1). ROC values were AUC 0.721 (0.118, 95\% CI: $0.490-0.952, p=0.031)$ and AUC $0.771(0.117,95 \%$ CI: $0.542-1.001, p=0.01)$, respectively for determining upon PSAD and mpMRI. The cut-off value was chosen to maximize both sensitivity and specificity, by applying the Youden index $($ maximum $=$ sensitivity + specificity -1$)$ for each ROC analysis (Table 3).
Table 3. Results of ROC analysis for $\mathrm{mpMRI}$ and PSAD

\begin{tabular}{lcccc} 
Characteristic & AUC & SE & Sensitivity & Specificity \\
\hline mpMRI & 0.771 & 0.117 & 0.875 & 0.656 \\
\hline PSAD & 0.721 & 0.118 & 0.875 & 0.75 \\
\hline PSA & 0.652 & 0.122 & 0.75 & 0.594
\end{tabular}

Cancer detection and Gleason score differentiation via mpMRI resulted in the initiation of therapy: i) radical prostatectomy (1 patients); ii) radiotherapy treatment - 

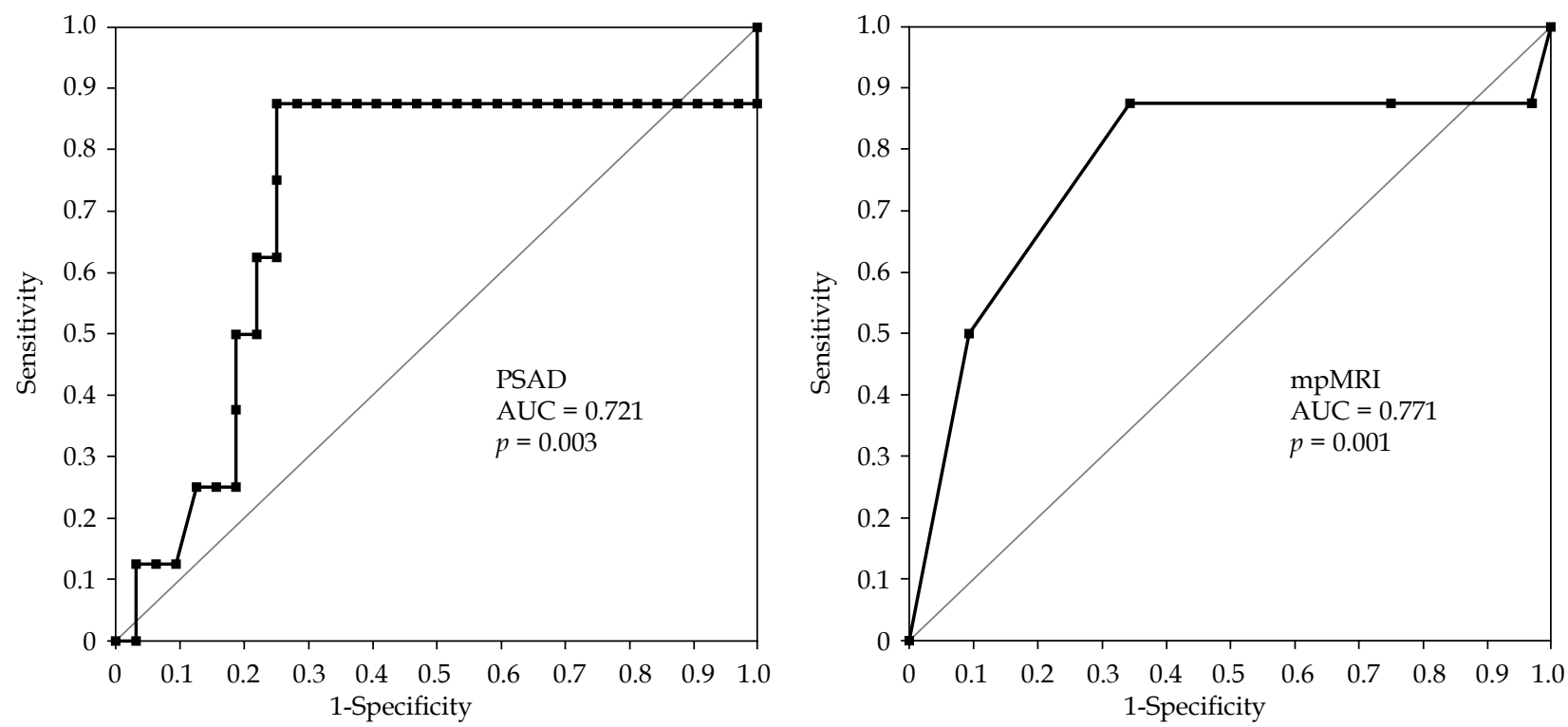

Fig. 1. Receiver operating curve (ROC) curve analysis with the use of mpMRI and PSAD

brachytherapy (6 patients); and iii) active surveillance for low-risk disease (1 patient). The obtained characteristics of the prostate cancers among the majority of our patients implied the use of brachytherapy [1] as the primary treatment method. Patients' preferences, when making a therapeutic decision and choosing the treatment method, were taken into account.

Prostate mpMRI PIRADS 1-2 reduces the need for repetitive biopsy through non-invasive serial monitoring for those under active surveillance.

\section{Discussion}

Diagnosis of clinically significant disease can be challenging even with application of state-of-art clinical methods. Biopsy remains the standard tool for PCa diagnosis. In prostate cancer diagnostics, standard TRUS$\mathrm{Bx}$ is characterized by low detectability (approximately $25 \%$ ), as three out of four patients undergoing biopsy are identified with no PCa [11]. As a consequence, a substantial number of patients continue to be under suspicion of cancer despite multiple benign biopsies. As a result, mpMRI is increasingly used to localize suspicious areas that could be targeted by MRI-TBx (MRI-targeted biopsy). To standardize mpMRI evaluation and decrease reporting ambiguity, the PI-RADS classification was created. The PIRADS v. 2 protocol is routinely used in the assessment of focal prostatic lesions. However, variability in interpretation of prostate mpMRIs, including PI-RADS, among radiology experts and novices, remains an ongoing problem [12]. This is reflected in the creation of subsequent versions of the PIRADS and conducting scientific research in order to assess the diagnostic efficiency of mpMRI [13].

MRI-guided biopsy (MRI-TBx) can be performed using different techniques. Three techniques of MRI-guided procedures are available: i) in-bore MRI; ii) MRI-ultrasound software-assisted fusion biopsy; and iii) cognitive biopsy [11]. Importantly, the experience of the biopsy operator plays a major role in the precision of targeted biopsies. The cognitive approach is simple but mostly operator dependent. It is imperative for the operator to have a good understanding of the position of the lesion in the prostate gland to make a precise biopsy. Despite this, the advantages of MRI-targeted biopsy are twofold: improving detection of high-grade cancer by reducing the false-negative rate of biopsy, and avoiding detection of low-grade disease by selectively targeting tumor foci which are more likely to be clinically significant [14]. Stratification of risk for PCa patients is of great importance, as the mortality of PCa greatly varies according to various factors and in particular Gleason score. Over the last decade, PCa screening (PSA, systematic biopsies) has reduced the incidence of advanced PCa and PCa mortality; however, it leads to over-diagnosis and over-treatment of low-risk and indolent PCA [11,15].

mpMRI performed in our study group allowed $60 \%$ of patients to avoid undergoing invasive biopsies and experiencing potential minor complications (hematuria, hematospermia, rectal bleeding, mild to moderate degrees of vasovagal episodes, genitourinary tract infection) and major complications (urosepsis, rectal bleeding requiring intervention, acute urinary retention, Fournier's gangrene and myocardial infarction) $[16,17]$. TRUS$\mathrm{Bx}$ is associated with significant pain and discomfort in a proportion of men and it has psychological effects, including anxiety and depression [17]. As the mortality of PCa greatly varies according to several factors and in particular the Gleason score, it is essential to distinguish types of $\mathrm{PCa}$ and adapt proper treatment. These patients are under constant observation with active monitoring.

MRI-TBx is shown to detect a significantly higher rate of International Society of Urological Pathology (ISUP) grade $>2$ cancers [18] and ISUP grade $>3[19,20]$ in comparison with standard TRUS-Bx. Among the men with prior negative biopsies, the advantage of MRI-targeted biopsy application in identifying areas of suspicion within the prostate, which as several recent series have demon- 
strated might have been overlooked during consecutive systematic sampling, is indisputable. It has been observed that targeted biopsy detected more clinically significant cancers and fewer clinically insignificant cancers than systematic biopsy [21]. In our study, eight out of ten performed TRUS-Bx biopsies confirmed PCa with Gleason score $\geq 6$ disease. Also Mendhiratta et al. found MRI fusion targeted biopsy to detect more Gleason score $\geq 7$ disease [22]. In the light of evidence suggesting a clinical benefit of targeted biopsy for men with prior negative biopsies, the National Comprehensive Cancer Network (NCCN) guidelines for prostate cancer detection have suggested that men with prior negative biopsies (two or more) who are subjected to repeat biopsy should undergo pre-biopsy mpMRI to identify the areas of occult disease [23]. csPCa diagnosis constantly evolves to develop the most effective methods, and the 2019 edition of the European Association of Urology (EAU), European Association of Nuclear Medicine (EANM), European Society for Radiotherapy and Oncology (ESTRO), European Society of Urogenital Radiology (ESUR), International Society of Geriatric Oncology (SIOG) guideline on prostate cancer recommends performing mpMRI before the first set of biopsies [24,25].

Analysis of Spearman's coefficient showed the highest statistical significance of correlation between mpMRI results and Gleason score in the group of PCa patients $\left(r_{s}=0.41, p=0.0085\right)$. This indicates the dependence between PI-RADS and Gleason scores. ROC analysis shows that the PSAD parameter has a higher diagnostic value in comparison with PSA (Table 3).

The low number of participants was due to the fact that they came from a single urological office, which is undoubtedly the limitation of this study. However, statistical analysis was carried out thoroughly and statistical significance was demonstrated in presented cases. We are still collecting data as new patients are referred for prostate biopsy and more physicians start to use pre-biopsy mpMRI. Additionally, based on the obtained mpMRI results, we will evaluate the significance of the SelectMDx test as a diagnostic tool to determine whether a patient is at higher or lower risk for prostate cancer and which patient can safely avoid biopsy.

\section{Conclusions}

Introducing pre-biopsy mpMRI into our contemporary $\mathrm{PCa}$ diagnosis pathway increased the diagnostic yield of transrectal biopsy by increasing the prostate cancer detection. This enabled the introduction of treatment (brachytherapy and radical prostatectomy) among patients with csPCa. mpMRI application also allowed biopsy to be avoided among patients with no csPCa.

\section{Disclosure}

The authors report no conflict of interest.

\section{References}

1. Roobol MJ, Steyerberg EW, Kranse R et al. A risk-based strategy improves prostate-specific antigen-driven detection of prostate cancer. Eur Urol 2010; 57: 79-85.
2. Bjurlin MA, Carter HB, Schellhammer P et al. Optimization of initial prostate biopsy in clinical practice: sampling, labeling and specimen processing. J Urol 2013; 189: 2039-2046.

3. Bjurlin MA, Meng X, Le Nobin J et al. Optimization of prostate biopsy: the role of magnetic resonance imaging targeted biopsy in detection, localization and risk assessment. J Urol 2014; 192: 648-658.

4. Siddiqui MM, Rais-Bahrami S, Turkbey B et al. Comparison of $\mathrm{MR} /$ ultrasound fusion-guided biopsy with ultrasound-guided biopsy for the diagnosis of prostate cancer. JAMA 2015; 313: 390-397.

5. Meng X, Rosenkrantz AB, Mendhiratta N et al. Relationship between prebiopsy multiparametric magnetic resonance imaging (MRI), biopsy indication, and MRI-ultrasound fusion-targeted prostate biopsy outcomes. Eur Urol 2016; 69: 512-517.

6. Ash D, Flynn A, Battermann J et al. ESTRO/EAU/EORTC recommendations on permanent seed implantation for localized prostate cancer. Radiother Oncol 2000; 57: 315-321.

7. Fredman ET, Traughber BJ, Gross A et al. Comparison of multiparametric MRI-based and transrectal ultrasound-based preplans with intraoperative ultrasound-based planning for low dose rate interstitial prostate seed implantation. J Appl Clin Med Phys 2019; 20: 31-38.

8. Shaaer A, Davidson M, Semple M et al. Clinical evaluation of an MRI-to-ultrasound deformable imageregistration algorithm for prostate brachytherapy. Brachytherapy 2019; 18: 95-102.

9. Weinreb JC, Barentsz JO, Choyke PL et al. American College of Radiology. PI-RADS Prostate Imaging - Reporting and Data System: 2015, Version 2. Eur Urol 2016; 69: 16-40.

10. Kryvenko ON, Epstein JI. Improving the evaluation and diagnosis of clinically significant prostate cancer. Curr Opin Urol 2017; 27: 191-197.

11. Mottet N, Bellmunt J, Bolla $M$ et al. EAU-ESTRO-SIOG guidelines on prostate cancer. Part 1: screening, diagnosis, and local treatment with curative intent. Eur Urol 2017; 71: 618-629.

12. Wang NN, Fan RE, Ghanouni P et al. Teaching Urologists "How to Read Multi-Parametric Prostate MRIs Using PIRADSv2": Results of an iBook Pilot Study. Urology 2019; 131: 40-45.

13. Mason J, Adiotomre E, Bownes $P$ et al. Importance of dynamic contrast enhanced magnetic resonance imaging for targeting biopsy and salvage treatments after prostate cancer recurrence. J Contemp Brachytherapy 2018; 10: 570-572.

14. Mendhiratta N, Rosenkrantz AB, Meng X et al. Magnetic resonance imaging-ultrasound fusion-targeted prostate biopsy in a consecutive cohort of men with no previous biopsy: reduction of over-detection through improved risk stratification. J Urol 2015; 194: 1601-1606.

15. Stark JR, Perner S, Stampfer MJ et al. Gleason score and lethal prostate cancer: does 3+4=4+3? J Clin Oncol 2009; 27: 3459-3464.

16. Efesoy O, Bozlu M, Çayan S et al. Complications of transrectal ultrasound-guided 12-core prostate biopsy: a single center experience with 2049 patients. Turk J Urol 2013; 39: 6-11.

17. Minervini A, Vittori G, Siena G et al. Morbidity and psychological impact of prostate biopsy: the future calls for a change. Asian J Androl 2014; 16: 415-417.

18. Kasivisvanathan, V, Rannikko AS, Borghi M et al. MRI-targeted or standard biopsy for prostate-cancer diagnosis. N Engl J Med 2018; 378: 1767-1777.

19. Rouvière $\mathrm{O}$, Puech $\mathrm{P}$, Renard-Penna $\mathrm{R}$ et al. Use of prostate systematic and targeted biopsy based on multiparametric MRI in biopsy-naive patients (MRI-FIRST): a prospective multicentre paired diagnostic study. Lancet Oncol 2019; 20: 100-109. 
20. Van der Leest M, Cornel E, Israël B et al. Head-to-head comparison of transrectal ultrasound-guided prostate biopsy versus multiparametric prostate resonance imaging with subsequent magnetic resonance-guided biopsy in biopsy-naive men with elevated prostate-specific antigen: a large prospective multicenter clinical study. Eur Urol 2018; 75: 570-578.

21. Sonn GA, Chang E, Natarajan $\mathrm{S}$ et al. Value of targeted prostate biopsy using magnetic resonance-ultrasound fusion in men with prior negative biopsy and elevated prostate-specific antigen. Eur Urol 2014; 65: 809-815.

22. Mendhiratta N, Meng X, Rosenkrantz AB et al. Prebiopsy MRI and MRI-ultrasound fusion-targeted prostate biopsy in men with previous negative biopsies: impact on repeat biopsy strategies. Urology 2015; 86: 1192-1198.

23. Carroll PR, Parsons JK, Andriole G et al. Prostate cancer early detection, version 1.2014. Featured updates to the NCCN Guidelines. J Natl Compr Canc Netw 2014; 12: 1211-1219.

24. Rouvière O, Schoots IG, Mottet N. Multiparametric magnetic resonance imaging before prostate biopsy: a chain is only as strong as its weakest link. Eur Urol 2019; 75: 889-890.

25. Cuocolo R, Stanzione A, Ponsiglione A et al. Clinically significant prostate cancer detection on MRI: a radiomic shape features study. Eur J Radiol 2019; 116: 144-149. 